\title{
Reactividad vascular in vitro y estudio morfométrico de venas safenas utilizadas como bypass coronario: técnica "no-touch" versus convencional
}

\author{
Manuel Quiroz F. ${ }^{1}$, Nicolás Drolett SF. ${ }^{2}$, Paulina Aguirre P. ${ }^{2}$, Fredi Cifuentes J. ${ }^{2}$, \\ Edgardo Mancilla S. ${ }^{3}$, Andrés Pumarino M. ${ }^{3}$ y Javier Labbé Z. ${ }^{3}$
}

In vitro vascular reactivity and morphometric study of saphenous veins graft used as coronary bypass: "no-touch" versus conventional technique

Introduction: Prolonging of the grafts permeability used in coronary bypass is a constant challenge. Objective: To compare anatomical and functional human saphenous veins (VSH) extracted "No touch" (NT) technique vs conventional technique (TC). Materials and Methods: Experimental study. VSH dissected with CT and NT in the Regional Hospital of Antofagasta cardiac surgery ward. VSH samples were sectioned into $3 \mathrm{~mm}$ rings and preserved in isolated organs chambers with Krebs-Ringer solution. To evaluate the vasomotor activity, norepinephrine $\left(10^{-6} \mathrm{M}\right)$, papaverine $\left(10^{-4} \mathrm{M}\right)$, acetylcholine $\left(10^{-6} \mathrm{M}\right)$ and sodium nitroprusside $\left(10^{-5} \mathrm{M}\right)$ was administered. A segment of samples was fixed in $10 \%$ formalin, processed and histological analyzed under light microscopy technique with hematoxylin-eosin, Verhoeff and orceína. Statistical analysis was performed using the Prism software Graphad. Results: Vascular Reactivity: norepinephrine-induced vasoconstriction was significantly higher in the group rings NT $v s$ TC $(\mathrm{p}<0.0001)$. Vasodilation was higher with papaverine and acetylcholine in the NT group $(\mathrm{p}<0.004)$ and $(\mathrm{p}<0.0003)$, respectively. Morphometric study: The NT group presented muscularis $(0.755$ vs 0.680 $\mathrm{mm})$, adventitious $(0.5600$ vs $0.4663 \mathrm{~mm})$, and total wall $(1.344$ vs $0.962 \mathrm{~mm})$ thicker than the TC group. No significant differences in vasa vasorum number identified. Conclusion: The NT group vasoconstrictor and vasodilator responds significantly better. Results correlate with morphometric differences.

Key words: saphenous vein; coronary artery bypass; pedicled 'no-touch'; vascular damage.

\section{Resumen}

Introducción: Prolongar la permeabilidad de los injertos utilizados en bypass coronario es un desafío constante. Objetivo: Comparar anatomofuncionalmente venas safenas humanas (VSH) extraídas con técnica convencional (TC) vs técnica "no-touch" (NT). Material y Método: Estudio experimental. Se diseccionó VSH con TC y NT en el pabellón de cirugía cardiaca del Hospital Regional de Antofagasta. Las muestras de VSH fueron seccionadas en anillos de $3 \mathrm{~mm}$ y conservados en cámaras de órganos aislados con solución Ringer-Krebs. Para evaluar la vasomotilidad se administró norepinefrina $\left(10^{-6} \mathrm{M}\right)$, papaverina $\left(10^{-4} \mathrm{M}\right)$, acetilcolina $\left(10^{-6} \mathrm{M}\right)$ y nitroprusiato de sodio $\left(10^{-5} \mathrm{M}\right)$. Un segmento de las muestras fue fijado en formalina al $10 \%$, procesado con técnica histológica y analizado bajo microscopia óptica. Las muestras fueron teñidas con hematoxilina-eosina, Verhoeff y orceína. El análisis estadístico fue realizado mediante el software Prism Graphad. Resultados: Reactividad vascular: La vasoconstricción inducida por noradrenalina fue significativamente superior en anillos del grupo NT vs TC ( $<<0,0001)$. La vasodilatación producida por papaverina y acetilcolina fue superior en el grupo NT $(p<0,004)$ y $(p<0,0003)$, respectivamente. Estudio morfométrico: El grupo NT presentó túnica muscular $(0,755$ vs $0,680 \mathrm{~mm})$, adventicia $(0,5600$ vs $0,4663 \mathrm{~mm})$ y pared total $(1,344$ vs $0,962 \mathrm{~mm})$ más gruesa que el grupo TC. No hubo diferencias significativas respecto el número de vasa vasorum. Conclusión: El grupo NT responde significativamente mejor a estímulos vasoconstrictores y vasodilatadores. Los resultados se asocian con las diferencias morfométricas.

Palabras clave: vena safena; bypass coronario; vena no-touch; daño vascular. 


\section{Introducción}

Sin duda la cirugía de bypass coronario es una de las más frecuentes dentro de la cirugía cardiaca y mejorar la eficacia de las técnicas utilizadas representa un desafío permanente. Para este procedimiento, el injerto más frecuente es la vena safena externa debido a su disponibilidad y fácil extracción ${ }^{1 .}$ Sin embargo, esta técnica reporta cifras de oclusión que van desde un $15 \%$ hasta un $30 \%$ el primer año poscirugía, pudiendo llegar a $50 \%$ a los 10 años ${ }^{2,3}$. Este bajo tiempo de permeabilidad se explicaría por el desarrollo de esclerosis venosa en la anastomosis venosa-arterial, la cual comienza desde el momento de la cirugía ${ }^{4}$. Otra alternativa para revascularización coronaria son los puentes arteriales ${ }^{2}$. Geha et al $(1974)^{5}$ demostró mediante evaluación angiográfica de 85 pacientes operados con injerto de vena safena que el $87 \%$ permanecía permeable luego de 2 años. Con el objetivo de superar esta cifra realizaron puentes con arteria mamaria interna como recurso alternativo, luego de dos años la permeabilidad fue del $99 \%$ en 133 pacientes 5 . Para buscar mejorías respecto a la vena safena se han utilizado arterias como la radial, epigástrica inferior, esplénica y subescapular, siendo la arteria mamaria interna el vaso de elección demostrando una permeabilidad de un 99\%-100\% a los 5 años en implante in situ .

Basado en antecedentes sobre las características funcionales del tejido adiposo perivascular, De Souza y colaboradores en 1996 describieron la técnica "no-touch" para extracción de la vena safena ${ }^{2}$. Esta consiste en retirar el injerto con el tejido adiposo adyacente, facilitando la extracción, disminuyendo la manipulación y el riesgo de dañar las túnicas vasculares ${ }^{7}$. Este simple procedimiento no requiere de una dilatación venosa, lo que disminuye aún más la posibilidad de daño ${ }^{7,8}$.

Desde la publicación de la técnica muchos estudios han avalado sus ventajas sobre la técnica convencional ${ }^{1,9-12}$. A pesar de esto, los mecanismos fisiopatológicos involucrados en la mayor permeabilidad obtenida con esta técnica no son completamente conocidos ${ }^{2}$. Los factores que podrían explicar la mejor funcionalidad del injerto serían el menor trauma mecánico y la acción protectora del tejido adiposo adyacente; como inconveniente se encuentra la extensión de la insición para extraer el injerto y las posibilidades de complicación de esta herida operatoria ${ }^{2}$.

Nuestro objetivo fue comparar mediante la reactividad vascular in vitro y la histología de las venas safenas obtenidas con la técnica convencional y con la técnica "no-touch".

\section{Materiales y Métodos}

Estudio analítico-experimental en el que estudió la respuesta miogénica in vitro de venas safenas humanas sometidas a fármacos vasoconstrictores y vasodilatadores. Además, se realizó el análisis de preparados histológicos previamente estandarizados. Las venas safenas fueron extraídas de pacientes en el Hospital Regional de Antofagasta según la programación habitual del pabellón de cirugía cardíaca. El procedimiento quirúrgico fue realizado por el mismo cirujano en cada caso y los pacientes fueron aleatorizados a una técnica u otra mediante muestreo aleatorio simple, durante un período de seis meses a partir del 1 de noviembre de 2014 hasta el 1 de mayo de 2015. Se incluyeron 14 pacientes que aceptaron el consentimiento informado. Se contó con aprobación del Comité de Ética de la Universidad de Antofagasta y consentimiento informado por parte de los pacientes.

\section{Técnica quirúrgica "no-touch"}

Se realiza incisión con bisturí y disección del tejido adiposo hasta visualizar la vena safena. Se continúa la disección distal mediante incisiones segmentarias manteniendo un espesor de aproximadamente $2 \mathrm{~cm}$ del tejido adiposo adyacente con lo cual, la disección no requiere manipulación directa de la vena. Se ligan las ramas perforantes con clips a 0,5 $\mathrm{cm}$ de la vena. La extensión de la vena dependerá de los requerimientos y patología del paciente. Una vez retirado el injerto se seccionan $2 \mathrm{~cm}$ y se depositan en solución tamponada de fosfato (PBS) (Figura 1).

\section{Técnica quirúrgica convencional}

Se inicia de igual forma que en la técnica descrita anteriormente, pero la disección de la vena no incluye el tejido graso perivascular, por lo tanto, se realiza una disección de la vena manipulándola directamente. Una vez seccionada la vena se deposita en suero fisiológico y se inyecta solución fisiológica con jeringa de $10 \mathrm{ml}$ con el fin de comprobar su permeabilidad y distenderla. Del trozo de vena se secciona $2 \mathrm{~cm}$ aproximadamente para el estudio (Figura 2).

\section{Reactividad vascular in vitro}

La muestra obtenida en pabellón se traslada al laboratorio de Fisiología Experimental de la Universidad de Antofagasta en un recipiente estéril con solución PBS. Se expone a una mezcla gaseosa de $95 \% \mathrm{O}_{2}$ y $5 \% \mathrm{CO}_{2}$ mientras se realiza la disección de la misma en anillos de $3 \mathrm{~mm}$. Posteriormente, los anillos se conservan en cámaras para órganos aislados de $15 \mathrm{ml}$ con solución Ringer-Krebs a una 
ARTÍCULO ORIGINAL

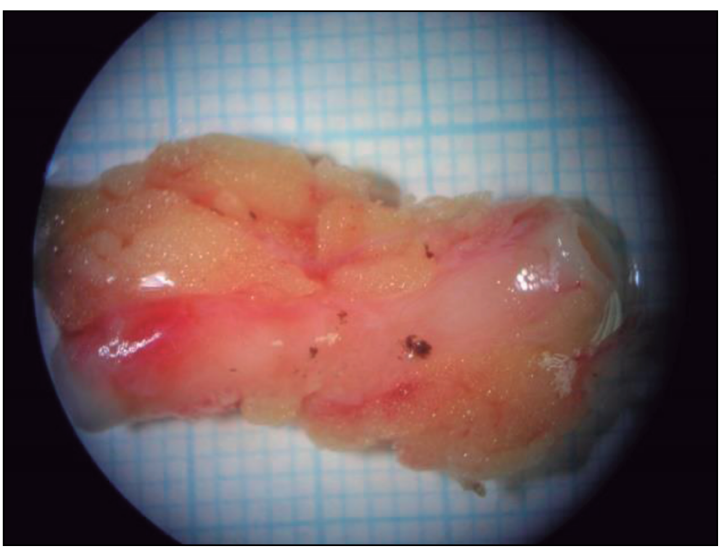

Figura 1. Vena safena extraída con técnica "no-touch" con abundante tejido adiposo perivascular.

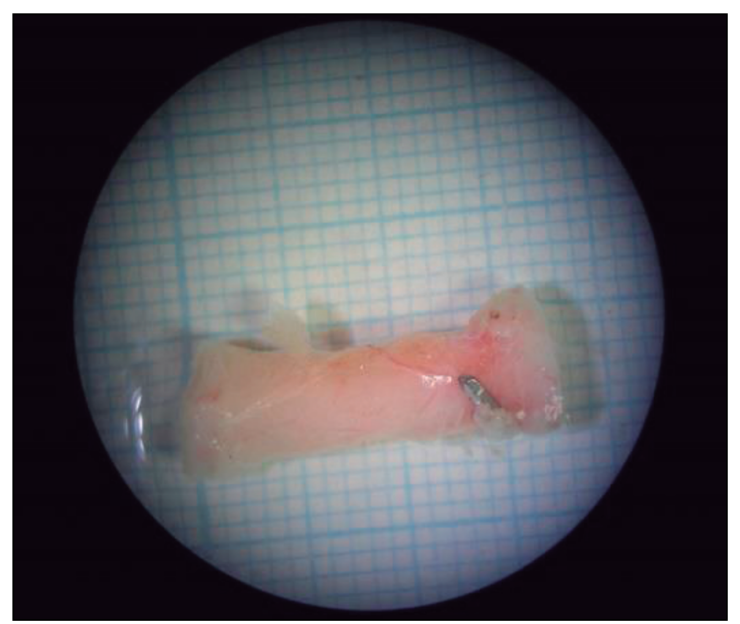

Figura 2. Vena safena extraída con técnica convencional.

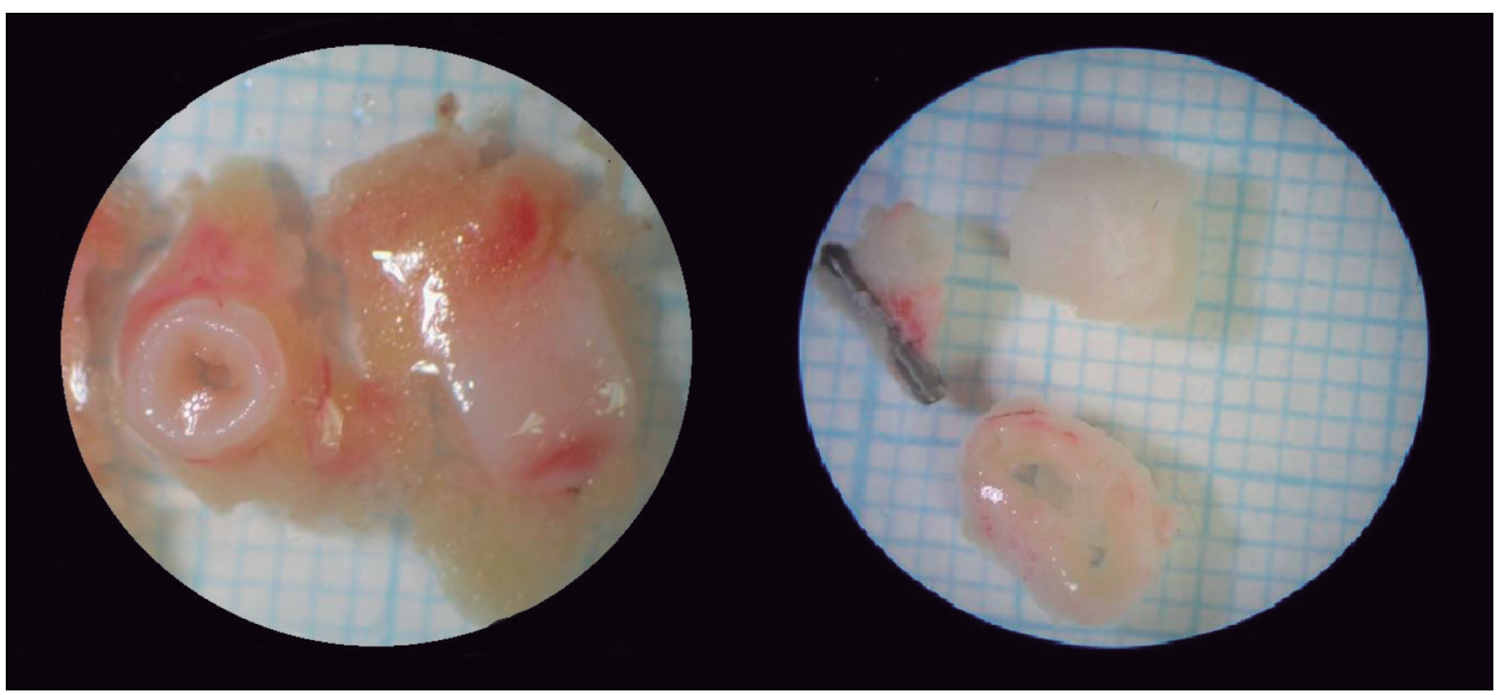

temperatura constante de $37^{\circ} \mathrm{C}$ y la misma mezcla gaseosa mencionada anteriormente. Los anillos son montados a través de 2 triángulos de acero inoxidable, uno fijando el anillo desde el extremo superior hasta el transductor de tensión y otro inferior, fijando el vaso al interior de la cámara de órganos aislados. La vasomotilidad se evaluó con la adición al baño de: norepinefrina $\left(10^{-6} \mathrm{M}\right)$, papaverina $\left(10^{-4}\right.$ $\mathrm{M})$, acetilcolina $\left(10^{-6} \mathrm{M}\right)$ y nitroprusiato $\left(10^{-5} \mathrm{M}\right)$, esperando su respuesta vasodilatadora durante 5 min. Previa a la aplicación de estos fármacos, se realizaron 3 curvas de $\mathrm{KCl} 60 \mathrm{mM}$ con ajuste de tensión a $1 \mathrm{~g}$. Las respuestas fueron obtenidas con transductores de tensión isométricos Radnoti ${ }^{\circledR}$, conectados a una unidad de adquisición de datos
PowerLab $8 / 35^{\circledR}$, expresadas en gramos, controladas e interpretadas con el software LabChart $7^{\circledR}$, que entrega las curvas respectivas (Figura 3 ).

\section{Estudio histopatológico}

La muestra obtenida de pabellón fue fijada inmediatamente con formalina al $10 \%$ y trasladada a la Unidad de Anatomía Patológica, donde se realizó técnica histológica y evaluación bajo microscopia óptica. Las muestras fueron teñidas con hematoxilina-eosina, verhoeff y orceína para visualizar las fibras elásticas. Se estudió el grosor de las túnicas vasculares, estado de fibras elásticas y número de vasa vasorum. Todas las muestras fueron analizadas por el mismo patólogo.
Figura 3. Anillos de 3 $\mathrm{mm}$ de venas extraídas con técnica no-touch (izquierda) y convencional (derecha). 


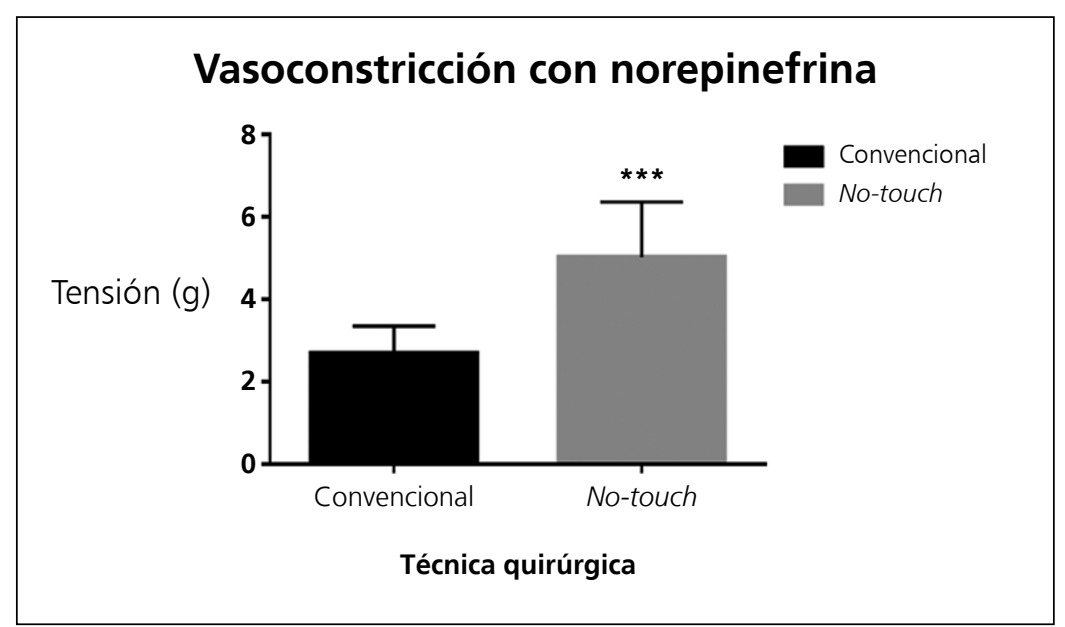

Figura 4. Comparación de la vasoconstricción a norepinefrina y promedio de cada grupo.

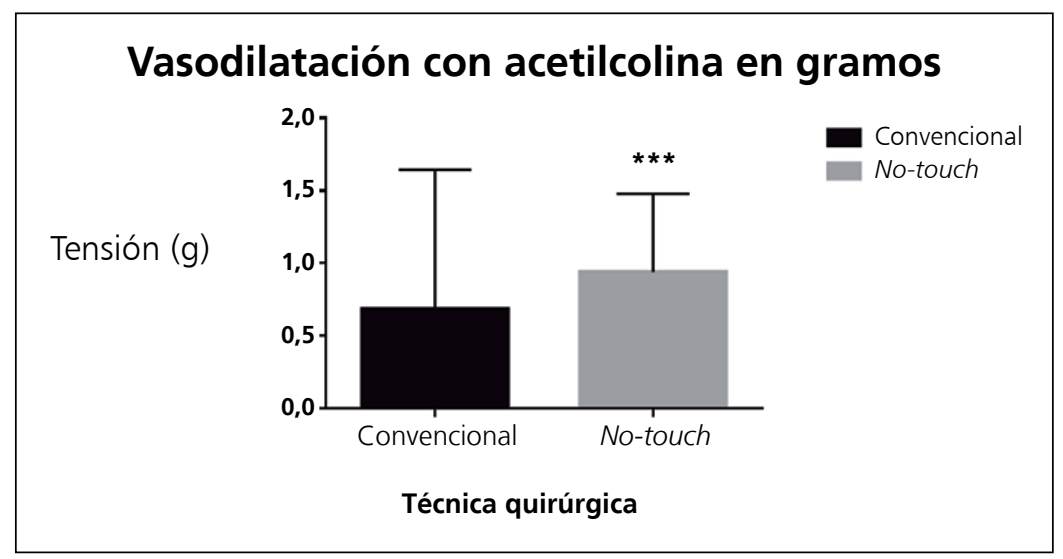

Figura 5. Comparación de la vasodilatación a acetilcolina y promedio de cada grupo.

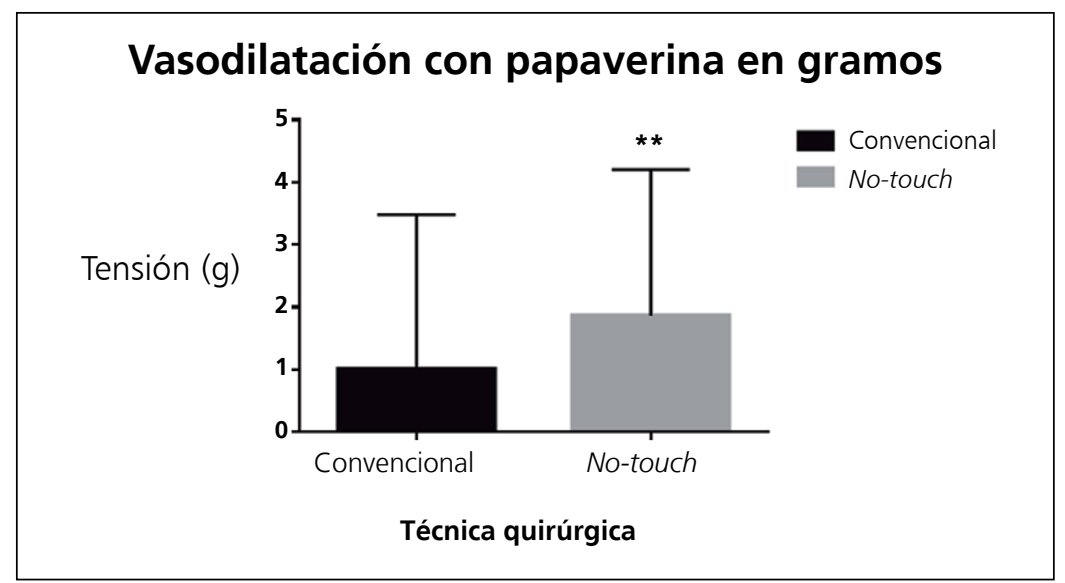

Figura 6. Comparación de la vasodilatación a papaverina y promedio de cada grupo.

\section{Análisis estadístico}

El análisis estadístico fue realizado mediante el software Prims Graphpad $8^{\circledR}$, en el cual se ha aplicado estadística descriptiva y analítica. Se realizó la prueba t para pruebas no paramétricas considerando significativo $\mathrm{p}<0,05$.

\section{Resultados}

\section{Reactividad Vascular in vitro}

Se estudiaron 89 anillos de VSH provenientes de los 14 pacientes, 57 del grupo NT y 32 del grupo TC. La vasoconstricción fue significativamente superior en anillos del grupo NT alcanzando una tensión media de $4.157 \mathrm{~g}$ vs $1.682 \mathrm{~g}$ del grupo TC $(\mathrm{p}=0,0001)$ (Figura 4). Respecto a la vasodilatación con acetilcolina (Figura 5), el grupo NT presentó una respuesta notablemente mejor con $(1.001 \mathrm{~g}$ vs $2.339 \mathrm{~g})$, siendo significativo $(\mathrm{p}=0,0003)$. La respuesta a la papaverina fue significativamente mayor en el grupo NT $(1,341 \mathrm{~g}$ vs $0,4517 \mathrm{~g} ; \mathrm{p}=0,004)$. (Figura 6). El efecto del nitroprusiato en ambos grupos fue una vasodilatación óptima con una tensión que llega a niveles basales. La reactividad se grafica en las Figuras 7 y 8.

\section{Estudio histopatológico}

Se extrajeron $16 \mathrm{VSH}, 8$ con TC y 8 con técnica NT. En la muestra no se rotuló la técnica quirúrgica. El grosor promedio de la túnica muscular (Figura 9) fue levemente menor en el grupo TC sin diferencias significativas con el grupo NT $(0,680 \mathrm{~mm}$ vs 0,775 $\mathrm{mm} ; \mathrm{p}=0,368)$. Respecto al grosor de la túnica adventicia, esta promedió $0,4663 \mathrm{~mm}$ en el grupo TC y $0,560 \mathrm{~mm}$ en el grupo NT $(\mathrm{p}=0,515)$ (Figura 10). El grosor total de la pared fue mayor en el grupo NT con $1.344 \mathrm{~mm}$ respecto del grupo TC, el cual tenía un grosor promedio de $0,962 \mathrm{~mm}(\mathrm{p}=0,131)$. Tampoco hubo diferencias significativas en el recuento de vasa vasorum (3,2 en el grupo TC vs 4,1 en el grupo NT). La fragmentación de las fibras elásticas fue similar en ambos grupos.

\section{Discusión}

Diferentes estudios in vitro, han mostrado una superioridad de la técnica NT en comparación a la $\mathrm{TC}$, sin embargo, aún no se han definido claramente los mecanismos que explican esta diferencia.

Durante la cirugía la obtención del injerto mediante la TC presenta un mayor riesgo de trauma, cuyos efectos en la permeabilidad a largo plazo no se conocen completamente, sin embargo, la disección, manipulación y dilatación de la vena a 
ARTÍCULO ORIGINAL

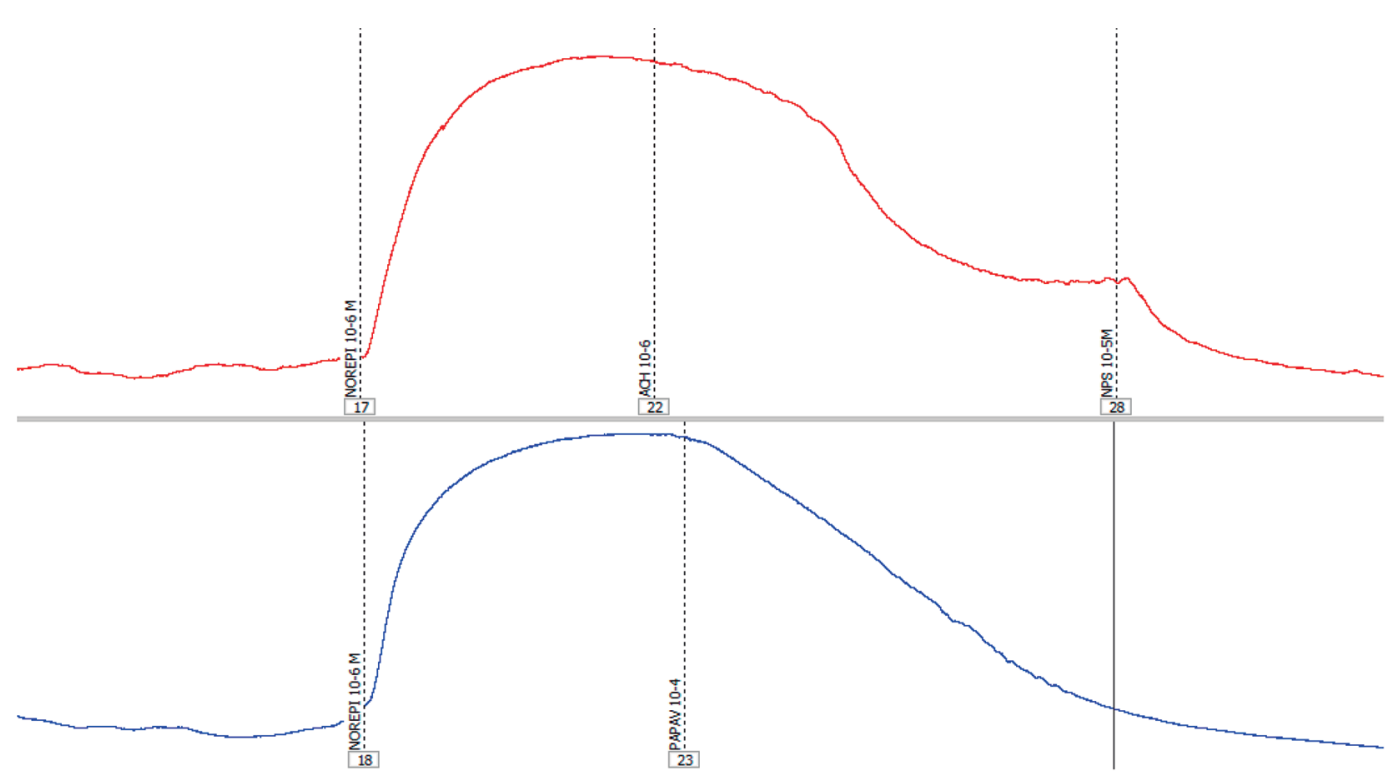

Figura 7. Gráfica representativa de reactividad vascular de vena extraída con NT. Rojo: Curva de registro con acetilcolina como vasodilatador (contracción máxima 6,7 g de tensión). Azul: Curva de registro con papaverina como vasodilatador (contracción máxima $5,0 \mathrm{~g}$ de tensión).

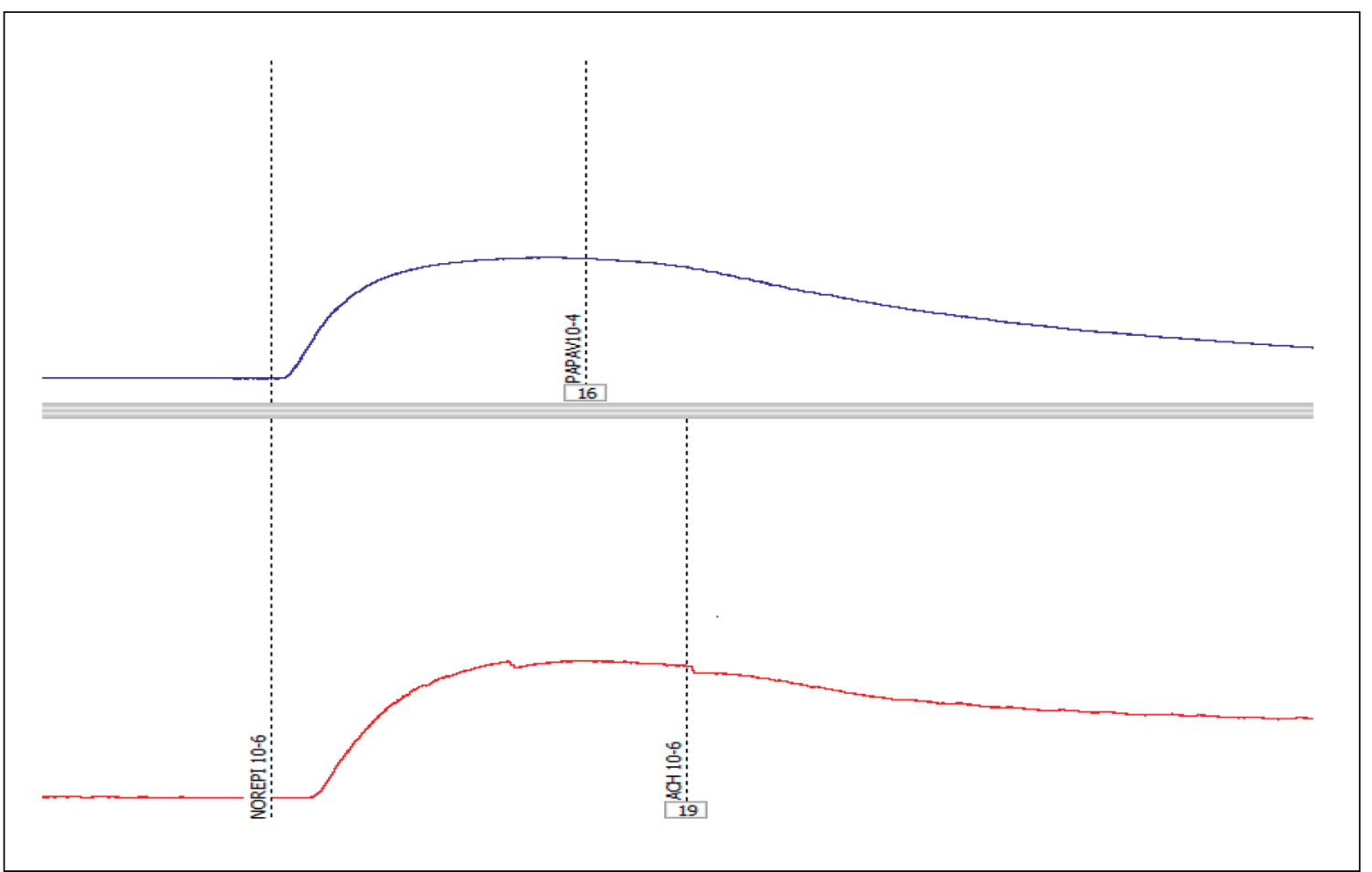

Figura 8. Gráfica representativa de reactividad vascular de vena extraída con TC. Azul: Curva de registro con papaverina como vasodilatador (contracción máxima 2,2 g de tensión). Rojo: Curva de registro con acetilcolina como vasodilatador (contracción máxima 2,0 g de tensión). 


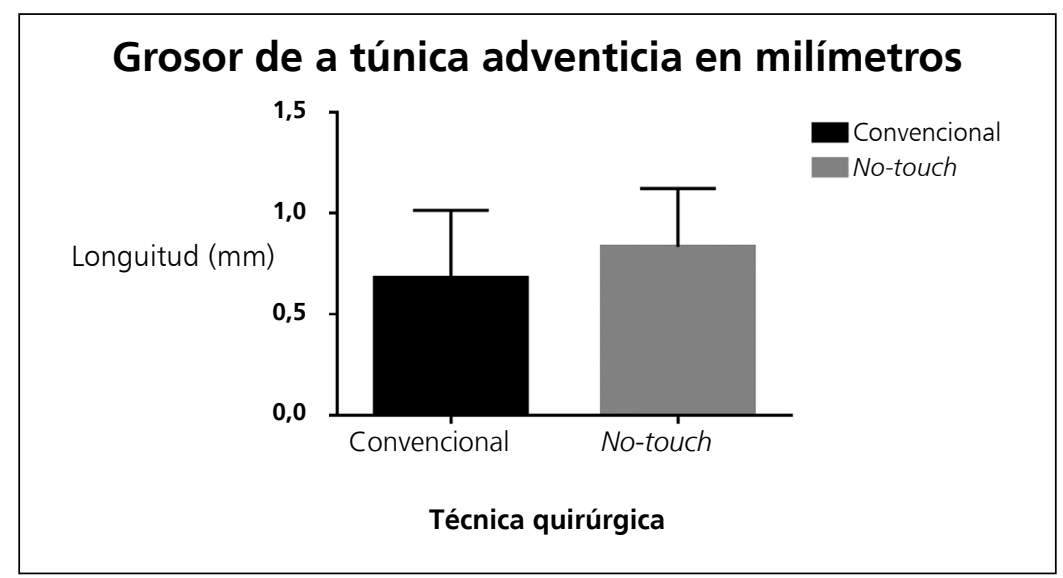

Figura 9. Espesor de la túnica muscular en micras de venas extraídas con NT y TC.

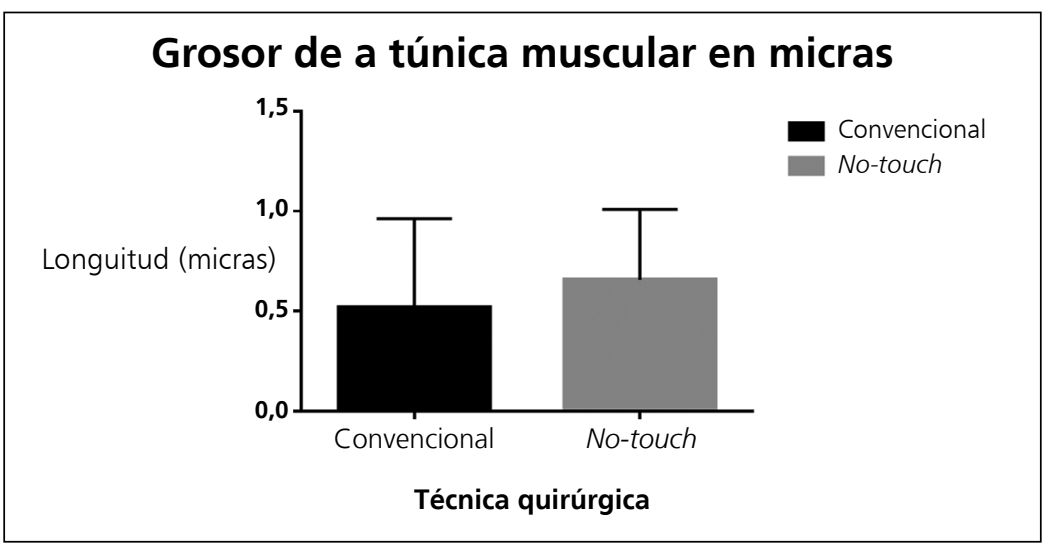

Figura 10. Espesor de la túnica adventicia de venas extraídas con NT y TC.

presiones de entre 350 y $700 \mathrm{mmHg}$, han mostrado denudamiento de la íntima y ruptura de la lámina elástica interna en estudios in vitro ${ }^{3,13-16}$.

Respecto al daño generado por la dilatación mecánica realizada en la TC, se ha reportado que el daño por la presión circunferencial ocasiona división celular atípica, colapso de los vasa vasorum u oclusión por trombos y deformación de las células endoteliales ${ }^{14}$. En nuestra experiencia, el recuento de vasa vasorum y el espesor de las túnicas íntima y media no presentó diferencias significativas.

La túnica adventicia presentó una ligera diferencia a favor de la técnica NT, lo que se explica en el menor trauma durante la disección, resultado que es compatible con lo reportado en otros estudios.

Esta alteración ha demostrado in vitro una relación directa con la presencia de hiperplasia neointimal y la consecuente falla del injerto ${ }^{17,18}$.

En relación a las diferencias funcionales en am- bas técnicas, estudios in vitro han identificado en la grasa perivascular y el tejido conectivo factores protectores para el injerto como la presencia de factores relajantes derivados de adipocitos (ADRFs) y el óxido nítrico $(\mathrm{ON})$ cuyos efectos vasodilatador, antitrombótico, antiinflamatorio y antiproliferativo son conocidos $^{19-23}$. Por el contrario, en la técnica convencional se encontró mayor activación y agregación plaquetaria, áreas de denudación endotelial con disminución de la producción de óxido nítrico sintasa y un aumento de factor de crecimiento celu$\operatorname{lar}^{6,15}$. Respecto a esto, nuestra experiencia mostró una diferencia significativa en relación a la capacidad vasodilatadora del injerto en la técnica NT, lo que podría ser el resultado de la acción de estos mediadores.

Sobre la respuesta vasoconstrictora la evidencia muestra una atenuación de la respuesta a norepinefrina ${ }^{27,28}$. Estos antecedentes no coinciden con nuestros resultados, ya que, encontramos una mejor acción contráctil al exponer los injertos NT a norepinefrina.

Se debe comprender que las ventajas de la técnica NT demostradas in vitro son de origen multifactorial, enfatizando en el menor daño del vaso en el proceso de extracción y la indemnidad endotelial que permiten una respuesta más fisiológica al ser implantado.

\section{Conclusiones}

Nuestro trabajo muestra que el grupo NT responde significativamente mejor a estímulos vasoconstrictores y vasodilatadores en comparación al grupo TC. Las diferencias en relación a la histología existen, sin embargo, no alcanzan diferencias estadísticamente significativas. Comprender las bases biológicas y moleculares involucradas en esta técnica es algo que aún está en desarrollo y permitirá decidir la mejor alternativa para nuestros pacientes.

\section{Agradecimientos}

Al equipo de Cirugía Cardíaca del Hospital Regional de Antofagasta, destacando a Raúl Contreras, Ruth Mamani Gómez, Pamela Aros Gonzales, Camila Azócar Cortés y Erica Escalante, por su compromiso constante y trabajo en equipo.

\section{Responsabilidades éticas}

Protección de personas y animales. Los autores declaran que para esta investigación no se 
han realizado experimentos en seres humanos ni en animales.

Confidencialidad de los datos. Los autores declaran que en este artículo no aparecen datos de pacientes.

Derecho a la privacidad y consentimiento in- formado. Los autores declaran que en este artículo no aparecen datos de pacientes.

Financiamiento: Sin financiamiento.

Conflicto de Intereses: No hay.

\section{Referencias}

1. Souza DS, Johansson B, Bojo L, Karlsson R, Geijer H, Filbey D, et al. Harvesting the saphenous vein with surrounding tissue for $\mathrm{CABG}$ provides long-term graft patency comparable to the left internal thoracic artery: results of a randomized longitudinal trial. J Thorac Cardiovasc Surg. 2006;132:373-8.

2. López-Jaramillo P, Bolívar IC, RuedaClausen CF, Calderón J, Castillo V, et al. Papel del tejido perivascular en la regulación del tono vascular: repercusión en el uso de puentes aorto-coronarios para revascularización miocárdica. Rev Colomb Cardiol. 2007;14:100-7.

3. Tsui JC, Souza DS, Filbey D, Karlsson MG, Dashwood MR. Localization of nitric oxide synthase in saphenous vein grafts harvested with a novel "no-touch" technique: potential role of nitric oxide contribution to improved early graft patency rates. J Vasc Surg. 2002; 35:35662.

4. Suma H. Arterial grafts in coronary bypass surgery. Ann Thorac Cardiovasc Surg. 1999; 5:141-5.

5. Geha AS, Krone RJ, McCormick JR, Baue AE. Selection of coronary bypass. Anatomic, physiological, and angiographic considerations of vein and mammary artery grafts. J Thorac Cardiovasc Surg. 1975;70:414-31.

6. Milesi V, Rebolledo A, Gómez Alvis A, Sanz N, Tommasi J, Adolfo Drago A, et al. Aspectos estructurales y funcionales de la vena safena humana utilizada como puente aorto-coronario en la cirugia de revascularizacion miocárdica. MEDICINA (Buenos Aires) 2001;61:481-90

7. Souza D. A new no-touch preparation technique. Technical notes. Scand J Thorac Cardiovasc Surg. 1996;30:41-4.

8. Souza DS, Bomfim V, Skoglund H, Dashwood MR, Borowiec JW, Bodin L, et al. High early patency of saphenous vein graft for coronary artery bypass harvested with surrounding tissue. Ann Thorac Surg. 2001;71:797-800

9. Souza DS, Dashwood MR, Tsui JC, Filbey D, Bodin L, Johansson B, et al. Improved patency in vein grafts harvested with surrounding tissue: results of a randomized study using three harvesting techniques. Ann Thorac Surg. 2002;73:1189-95.

10. Tsui JC, Souza DS, Filbey D, Bomfim V, Dashwood MR. Preserved endotelial integrity and nitric oxide synthase in saphenous vein grafts harvested by a 'notouch' technique. Br J Surg. 2001;88:1209-15.

11. Johansson B, Souza D, Bodin L. Slower progression of atherosclerosis in vein grafts harvested with 'no-touch' technique compared with conventional harvesting technique in coronary artery bypass grafting: an angiographic and intravascular ultrasound study. Eur J Cardiothorac Surg. 2010;38:414-9.

12. Dreifaldt M, Souza D, Loesch A. The 'no-touch' harvesting technique for vein grafts in coronary artery bypass surgery preserves an intact vasa vasorum. J Thorac Cardiovasc Surg. 2011;141:145-50.

13. Dashwood MR, Tsui JC 'No-touch' saphenous vein harvesting improves graft performance in patients undergoing coronary artery bypass surgery: A journey from bedside to bench; Vascular Pharmacology 2013;58:240-50.

14. Ahmed SR, Johansson BL, Karlsson MG, Souza DS, Dashwood MR, Loesch A. Human saphenous vein and coronary bypass surgery: ultrastructural aspects of conventional and "no-touch" vein graft preparations. Histol Histopathol. 2004;19:421-33.

15. Parish MA, Asai T, Grossi EA, Esposito R, Galloway AC, Colvin SB, et al. The effects of different techniques of internal mammary artery harvesting on sternal blood flow. J Thorac Cardiovasc Surg. 1992;104:1303-7.

16. Rosenfeldt FL, He GW. Buxton BF.
Pharmacology of coronary artery by- pass grafts. Ann Thorac Surg. 1999;67;878-88.

17. Loesch A, Dashwood MR. On the sympathetic innervation of the human greater saphenous vein: relevance to clinical practice. Curr. Vasc. Pharmacol. 2009;7;58-67.

18. Dreifaldt M, Souza DS, Loesch A, Muddle JR, Karlsson MG, Filbey D, Bodin L, et al. The "no-touch" harvesting technique for vein grafts in coronary artery bypass surgery preserves an intact vasa vasorum. J Thorac Cardiovasc Surg. 2011;141;14550. Síp?

19. Dashwood MR, Savage K, Dooley A, Shi-Wen X, Abraham DJ, Souza DS. Effect of vein graft harvesting on endothelial nitric oxide synthase and nitric oxide production. Ann Thorac Surg. 2005;80:939-44.

20. Dashwood M, Anand R, Loesch A, Souza D. Surgical trauma and vein graft failure: further evidence for a role of ET-1 in graft occlusion. J Cardiovasc Pharmacol. 2004;44: S16-S19.

21. Souza DS, Christofferson RH, Bomfim V, Filbey D. "No-touch" technique using saphenous vein harvested with its surrounding tissue for coronary artery bypass grafting maintains an intact endothelium. Scand Cardiovasc J. 1999;33:323-9.

22. Gollasch $M$. Vasodilator signals from perivascular adipose tissue. $\mathrm{Br} \mathrm{J}$ Pharmacol. 2012;165;633-42.

23. Maenhaut N, Van de Voorde J. Regulation of vascular tone by adipocytes. BMC Med. 2011;9;25. [i.

24. Lohn M, Dubrovska G, Lauterbach B, Luft FC, Gollasch M, Sharma AM. Periadventitial fat releases a vascular relaxing factor. FASEB J. 2002;16:105763.

25. Verlohren S, Dubrovska G, Tsang SY, Essin K, Luft FC, Huang Y, et al. Visceral periadventitial adipose tissue regulates arterial tone of mesenteric arteries. Hypertension 2004;44:271-6. 